\title{
A Novel Forwarding Paradigm for Position-Based Routing (with Implicit Addressing)
}

\author{
Holger Füßler*, Jörg Widmer ${ }^{\dagger}$, Martin Mauve ${ }^{\ddagger}$, and Hannes Hartenstein ${ }^{\S}$ \\ *Lehrstuhl für Praktische Informatik IV, University of Mannheim, Germany \\ ${ }^{\dagger}$ Federal Institute of Technology - Lausanne (EPFL), Switzerland \\ †University of Düsseldorf, Germany

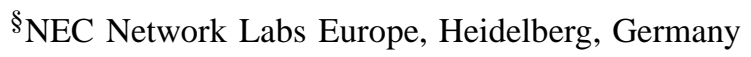

\begin{abstract}
Existing position-based unicast routing algorithms which forward packets in the geographic direction of the destination require that the forwarding node knows the positions of all neighbors in its transmission range. This information on direct neighbors is gained by observing beacon messages each node sends out periodically. Due to mobility, the information that a node receives about its neighbors becomes outdated, leading either to a significant decrease in the packet delivery rate or to a steep increase in load on the wireless channel as node mobility increases.

In this paper, we describe a mechanism to perform positionbased unicast forwarding without the help of beacons. In our contention-based forwarding scheme, the next hop is selected through a distributed contention process based on the actual positions of all current neighbors. For the contention process, CBF makes use of biased timers. To avoid packet duplication, the first node that is selected suppresses the selection of further nodes. Since the basic scheme can lead to packet duplication, we describe alternative ways of suppressing those. In addition to that, we compare the CBF schemes and standard greedy forwarding by means of simulation with ns- 2 .
\end{abstract}

\section{INTRODUCTION}

Although several decades old, the concept of position-based routing received renewed interest during the last few years as a method for routing in mobile ad-hoc networks [1], [2], [3], [4]. The general idea of position-based routing is to select the next hop of a packet based on position information such that the packet is forwarded in the geographical direction of the destination. This forwarding decisions is based purely on local knowledge. It is not necessary to create and maintain a "global" route from the sender to the destination. Therefore, position-based routing is commonly regarded as highly scalable and very robust against frequent topological changes.

A pre-requisite for position-based routing is to know the position of the destination of a packet. For this purpose, distributed algorithms called location services have been proposed. For the remainder of this paper, we assume that such an algorithm is in place.

In all existing strategies for greedy unicast forwarding, the position of a node is made available to its direct neighbors (i.e., nodes within single-hop transmission range) in form of periodically transmitted beacons. Each node stores the information it receives about its neighbors in a table and thus maintains position information about all direct neighbors. The state expires after a certain amount of time.

Given its own position, the "last-known" position of the direct neighbors, and the position of the destination of the packet, a node selects a next hop out of his neighbor table according to a forwarding strategy. One frequently used heuristic is picking the neighbor minimizing the remaining distance to the destination under the constraint that the neighbor has a smaller distance than the forwarding node. Once a neighbor is selected, it is addressed directly with its MAC address. This process is called 'greedy forwarding'. If a node with positive progress towards the destination does not exist, a recovery strategy(e.g. [1], [4], [5]) is used but for the remainder of this work we will focus on the greedy part of the forwarding.

Greedy forwarding faces the following drawbacks:

1) The position information of the neighbors looses accuracy over time in the presence of mobility. In the worst case a node that was reachable has moved out of range. Since in general radio links have a high error rate, this situation is often difficult to identify. Usually [6] it is accomplished by assuming that is node is unreachable if it did not acknowledge a packet after a certain number of retries. Thus, the inaccuracy of the neighbor information places additional load on the MAC layer and - if the routing algorithm can not react to this transmission failure - the packet is lost. If the algorithm reacts to the link failure by removing the faulty neighbor from the neighbor table and selecting another neighbor for forwarding, it avoids packet loss at the cost of additional packet delay (and the risk of choosing another unreachable node as forwarder).

2) The beacons themselves impose additional load on the network. The higher the frequency of beacons, the lower the aforementioned inaccuracy of neighbor information but the higher the load on the network. 
3) Since beacons are transmitted with link-layer broadcast, a transmission failure can not be detected, resulting in nodes being close and not recognized as being neighbors. This can lead to suboptimal forwarding decisions, the unnecessary initiation of the recovery procedure, or to packet loss. With a high node density, even increasing the beaconing frequency does not help much since the probability of beacon collisions increases as well.

4) The assumption of bi-directional links needed by neighbor-table-based forwarding is not necessarily true for real radio links.

In this paper, we describe a novel greedy forwarding strategy for position-based routing algorithms called ContentionBased Forwarding (CBF). CBF performs greedy forwarding without the help of beacons and without the maintenance of information about the direct neighbors of a node. Instead, all suitable neighbors of the forwarding node participate in the next hop selection process and the forwarding decision is based on the actual position of the nodes at the time a packet is forwarded. This is in contrast to existing greedy forwarding algorithms that base their decision on the positions of the neighbors as perceived by the forwarding node and eliminates the problems outlined above. In order to escape from local optima, existing recovery strategies can either be used directly or may be adapted to be used with $\mathrm{CBF}$.

The contention process of CBF used for next-hop selection represents a paradigm change in the forwarding of packets. In traditional protocols, the forwarder actively selects the desired next-hop by unicasting the packet to the corresponding MAC address. In contrast, with CBF the responsibility for next-hop selection lies with the set of possible next hops. Furthermore, if no other interaction between forwarder and next hop is required, which is the case for two of the three presented strategies, MAC layer addresses become obsolete.

CBF consists of two parts: the selection of the next hop is performed by means of contention, while suppression is used to reduce the chance of accidentally selecting more than one next hop. We present three suppression strategies with different characteristics. The results of our study show that suppression of duplicate packets works well, that CBF achieves similar packet delivery ratios as beacon-based greedy routing, and that it dramatically reduces the load on the wireless medium for a given delivery rate if node mobility is high. $\mathrm{CBF}$, therefore, represents a good alternative to traditional beaconbased greedy forwarding.

Similar protocols were proposed independently in the appendix of [7] and in [8]. A more detailed discussion of the topics covered in this paper including a more comprehensive list of related publications can be found in [9].

The remainder of this paper is structured as follows: Section II contains a description of $\mathrm{CBF}$ with three alternative suppression schemes. In Section III, CBF is compared to an existing greedy forwarding strategy by means of simulation. Finally, Section IV points out directions of future work and concludes the paper.

\section{CONTENTION-BASED ForwARDinG}

The general idea of CBF is to base the forwarding decision on the current neighborhood as it exists in reality and not as perceived by the forwarding node. This requires that all suitable neighbors of the forwarding node are involved in the selection of the next hop.

CBF works in three steps: first, the forwarding node transmits the packet as a single-hop broadcast to all neighbors. Second, the neighbors compete with each other for the "right" to forward the packet. During this contention period, a node determines how well it is suited as a next hop for the packet. Third, the node that wins the contention suppresses the other nodes and thus establishes itself as the next forwarding node.

In the following we describe in detail how contention can be realized on the basis of biased timers and present three alternative suppression strategies.

\section{A. Timer-Based Contention}

The timer-based contention scheme works as follows: A node $f$ that wishes to forward a packet simply broadcasts it (together with the node's own position and the position of the destination $z$ ). A node $n$ that receives the broadcast checks if its own position is closer to the destination than that of the broadcasting node. The packet progress (normalized to the radio range) is given by:

$$
P(f, z, n)=\max \left\{0, \frac{\operatorname{dist}(f, z)-\operatorname{dist}(n, z)}{r_{\text {radio }}}\right\}
$$

In the case of $P>0$, the $n$ sets a timer with expiry time $t(P)=T(1-P)$ where $T$ is the maximum forwarding delay. When this timer expires, node $n$ re-broadcasts the packet. The construction of the expiration time ensures that better suited forwarders re-broadcast earlier. Every node hearing the packet being forwarded by another node cancels its own timer. The timer setting represents a contention process (earliest forwarder wins), the cancellation of the timer suppresses of redundant retransmissions.

This simple method, called it 'basic suppression scheme', faces two problems: First, two potential forwarders could select two similar timeout values, resulting in one of the nodes not being able to cancel its transmission process (depending on the time it takes to do so). Second, two forwarders could be outside each others radio range, not being able to decode the packet by its competitor and thus forwarding it independently. While the former issue can be alleviated by increasing the maximum forwarding delay $T$ (more extensively discussed in[9]), the latter requires modifications to the protocol itself.

\section{B. Suppression}

Depending on where potential next hops are located, they may be out of transmission range and will thus not be be able to suppress each other. In the worst case, up to three copies of the packets may be forwarded, as shown in Figure 1. The larger the number of nodes within transmission range of the source, the higher the probability of one or more packet duplications. 


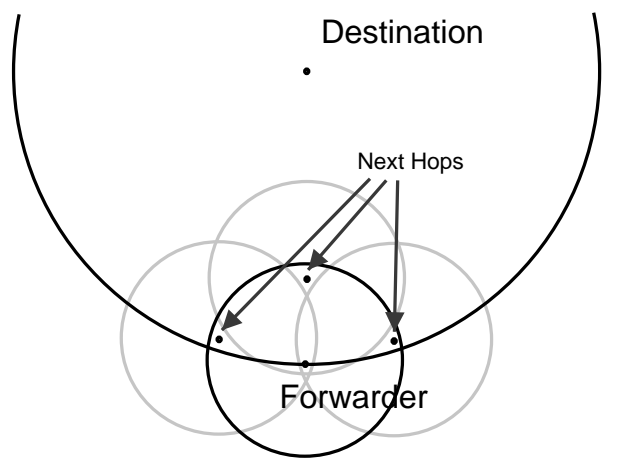

Fig. 1

PACKET DUPLiCATION IN THE BASIC SCHEME

1) Area-Based Suppression: In order to avoid the extra packet duplications from the basic suppression scheme we propose to artificially reduce the area from which the next hop is selected. We call this reduced area the suppression area and the algorithm area-based suppression. The key idea is to choose the suppression area such that all nodes within that area are in transmission range of each other, avoiding extra packet duplications as they may appear in the basic suppression scheme.

Area-based suppression requires a decision on how the suppression area is chosen. One possible choice is a circle with the diameter of the transmission range located within the forwarding node's transmission range in direction of the destination (e.g., the gray circle in Figure 2). A circle is the geometric shape covering the largest area given that any two points within the shape are no farther apart than the transmission range. If the nodes are uniformly distributed this means that on average the circle will contain the highest number of neighboring nodes when compared to other shapes where the distance between any two points does not exceed the transmission range. However, several parts of the forwarding area which make good forwarding progress are not included in the circle. A different shape where any two points are no further apart than the transmission range, the Reuleaux triangle [10], much better covers the area with good forwarding progress (see Figure 2). ${ }^{1}$ By using the Reuleaux triangle with a width of the transmission range, we trade off the number of nodes contained in the suppression area against the inclusion of better suited nodes. Between $60 \%$ and $100 \%$ forwarding progress with respect to the radio range, the Reuleaux triangle covers more of the neighbors than the circle and above approximately $80 \%$, the reuleaux triangle covers all of the neighbors with this progress. Therefore, it is more likely to include a node with good forwarding progress.

Given the Reuleaux triangle as suppression area, the suppression algorithm works as follows:

\footnotetext{
${ }^{1}$ A Reuleaux triangle with a width of $r$ can be constructed by placing three circles with radius $r$ at the corners of an equilateral triangle with an edge length $r$. The intersection of the circles is the Reuleaux triangle.
}

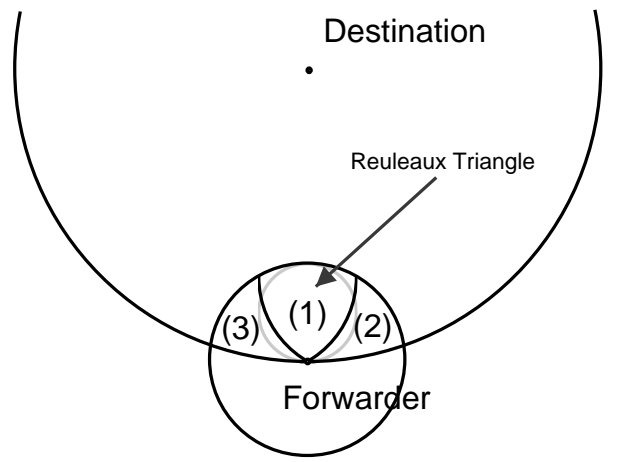

Fig. 2

FORWARDING AREAS

- The forwarding node broadcasts the packet.

- Only the nodes contained in the Reuleaux triangle participate in the contention process.

- The node at which the timer runs out first is the next hop and broadcasts the packet.

- All other nodes are suppressed. Packet duplication may occur only because of the time required for suppression (or because of node movement).

Of course it is possible that the only neighbors of the forwarding node that provide forward progress toward the destination are not contained in the Reuleaux triangle (1). In this case the forwarding node will not hear another node forwarding the packet. Consequently, the process is repeated with the remaining areas (2) and (3) where nodes with forwarding progress may be located, until the forwarding node hears a rebroadcast of the packet. If no node within areas (1), (2), or (3) responds, then there is no node with positive forward progress and a recovery strategy has to be used just like in existing position-based forwarding schemes. The order in which areas (2) and (3) are selected when no node is located in area (1) should be chosen randomly. This way, a tendency to always route around areas with little or no coverage in the same direction is avoided.

The key advantage of area-based suppression is the reduction of packet duplications. This comes at the cost of requiring up to three broadcasts for forwarding a packet. However, it is important to realize that requiring more than one broadcast becomes less and less likely as the number of nodes increases. Furthermore, the Reuleaux triangle covers the largest of the three areas and therefore has the highest probability of containing a potential next hop.

2) Active Selection: While area-based suppression eliminates the packet duplications caused by nodes not being in transmission range of each other it does not prevent packet duplications caused by the time required to perform the suppression. Active selection of the next hop prevents all forms of packet duplication at the cost of additional control messages. It is inspired by the Request To Send, Clear To Send (RTS/CTS) MACA-scheme proposed in [11] and used (as a variant) in IEEE 802.11 (see [6]). 
The scheme works as follows: the forwarding node broadcasts a control packet called RTF (Request To Forward) instead of immediately broadcasting the packet. The RTF contains the forwarding node's location and the final destination's location. Every neighbor checks if it provides forward progress for the packet announced by the RTF. If this is the case it sets a reply timer according to the basic suppression scheme. If the timer runs out, a control-packet called CTF (Clear To Forward) is transmitted to the forwarding node. The CTF packet contains the position of the node sending the CTF. If a node hears a CTF for the packet, it deletes its own timer and is suppressed.

The forwarding node may receive multiple CTF controlpackets. Of all neighbors that have transmitted a CTF packet it selects the node with the largest forward progress and transmits the packet to this node using unicast. An additional benefit of active selection compared to basic and areabased suppression is that it may be integrated with RTS/CTS schemes to alleviate the "hidden terminal problem".

Active selection prevents all forms of packet duplication, even though multiple nodes may send a CTF control packet. The forwarding node acts as a central authority deciding which node is selected as the next hop. This comes at the cost of additional overhead in form of RTF/CTF control packets.

\section{Protocol Simulations}

\section{A. Simulation Setup}

The proposed mechanisms were implemented in the network simulator [12] using the existing IEEE 802.11 MAC (with several bug-fixes). The size of the simulated area was $2 \mathrm{~km} \times 2 \mathrm{~km}$. We simulate different node densities and different levels of mobility using the Random Waypoint Model [13] modeling different mobility rates by adjusting the maximum speed with the pause time set to zero. The traffic pattern consists of 4 packets per second of size 128 bytes, where sender and receiver are selected randomly.

In the simulation, the three CBF schemes compete with two versions of beacon-based greedy forwarding. The first, which we call 'basic greedy', is not informed by the link-layer if a selected next-hop is not reachable and the packet is lost. With the 'optimized greedy' scheme, the link-layer has the ability to inform the routing layer if such an event occurs. The routing layer then removes the unreachable next-hop and attempts to reroute the packet.

Here, we only discuss the scenario where 300 nodes are distributed in the simulation area, since it has a reasonable likelihood that a greedy route to the destination exists. A more detailed description of simulation results is given in [9].

\section{B. Packet Delivery Ratio}

Figure 3 shows the packet delivery ratio of the three CBF schemes, the basic greedy scheme for three different beacon intervals (in seconds) and the optimized greedy scheme for a beacon interval of one second. The values for optimized greedy with other beacon intervals were omitted because their performance is similar to the one with a beacon interval of 1 second in the chosen scenarios. The $\mathrm{x}$-axis shows the four different groups of movement scenarios with their respective maximum node speed.

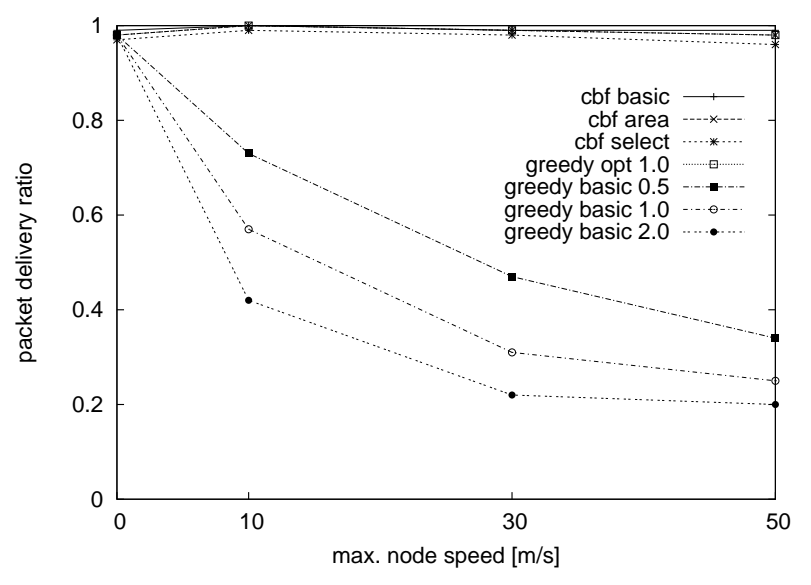

Fig. 3

PACKET DELIVERY RATIO (300 NODES / $4 \mathrm{KM}^{2}$ )

As can be seen from the graph, all CBF schemes and the optimized greedy scheme reach very high packet delivery ratios. Since the node density is fairly high, greedy routes exist most of the time. Only the packet delivery ratio of the active selection scheme suffers slightly when mobility is high. In such scenarios it is possible that a node moves out of transmission range before sending the CTF (which nevertheless may suppress the CTFs of other nodes) or before receiving the actual data packet. Currently, the active selection scheme uses no recovery strategy that attempts to retransmit a packet if no CTF is heard after the timeout interval $T$, and the packet is lost.

In contrast to the CBF schemes and to the optimized greedy approach, the basic greedy scheme performs significantly worse under mobility. With a maximum node speed of $50 \mathrm{~m} / \mathrm{s}$ the packet delivery ratio drops to 0.2 with a beacon interval of 2 seconds. 'Basic greedy' selects a greedy forwarder out of the list of neighbors and tries to transmit the packet to it. If a neighbor moves out of transmission range, its entry expires and it is removed from the neighbor table after a timeout period during which no packets are received. ${ }^{2}$ During this period, all packets handed down to the link layer with this node as next hop are lost. The optimized greedy scheme detects these failures and reroutes all packets in the MAC queue destined for this next hop. Consequently, no packets are lost when the best suitable neighbor leaves the radio range if there is another suitable next hop in the neighbor table. The higher the node mobility, the more packets cannot by delivered with the basic greedy scheme and are therefore re-queued by the optimized scheme. Hence, the good performance of the optimized scheme comes at the expense of a trial-anderror strategy to detect a suitable forwarder that is still in

\footnotetext{
${ }^{2}$ This beacon expiry timeout is usually a multiple of the beacon interval. We chose it as 3.5 times the beacon interval as in the simulations in [1].
} 
transmission range, which may significantly increase the per hop delay and the network load. The CBF schemes achieve similar packet delivery ratios without any link layer packet loss recovery for the packet transmissions.

\section{Transmission Costs}

In Figure 4 we show the transmission cost for the optimized greedy schemes and the CBF mechanisms in terms of average number of bytes transmitted at the MAC layer over the course of the simulation. The basic greedy schemes were omitted for lack of comparability; at high mobility, the packet delivery ratio is too low to allow a meaningful interpretation of the total overhead.

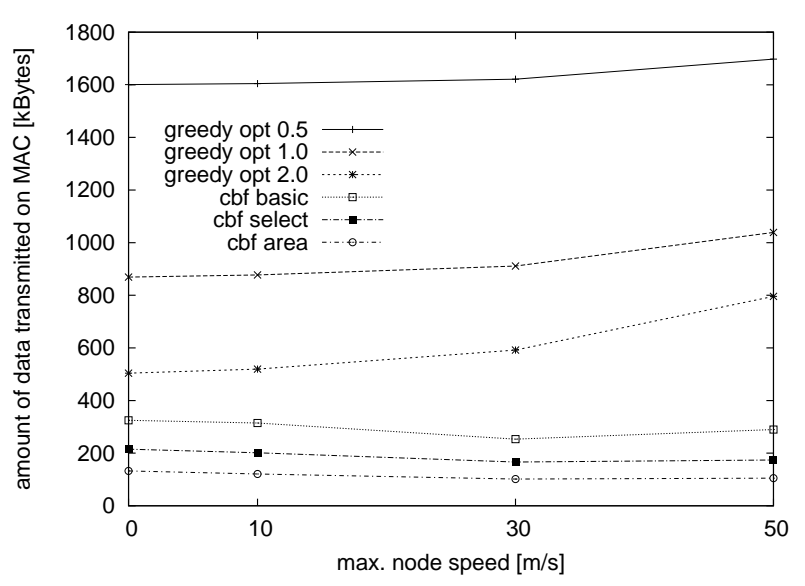

Fig. 4

TRANSMISSION COSTS ON MAC LAYER (300 NODES / $4 \mathrm{KM}^{2}$ )

As expected, all CBF methods use less bandwidth than the greedy schemes together with the overhead caused by the beacon messages. ${ }^{3}$ The area-based scheme consumes the least bandwidth, as no packet duplication occurs and - given a node density of approximately 15 nodes within transmission range - the forwarding node is almost always located within the Reuleaux triangle. Active selection causes a slightly higher overhead through the additional RTF and CTF messages and the basic CBF schemes causes the highest transmission costs due to packet duplication. The bandwidth consumption of all CBF schemes is relatively independent of mobility. The slight decrease in overhead can mainly be attributed to the decrease in the average path length caused by the random waypoint model.

The overhead caused by optimized greedy routing depends on a number of factors. The amount of data transmitted for beacon messages scales proportionally to the number of nodes, the beacon interval, and the simulation time. The value decreases somewhat with an increase in traffic since implicit beaconing causes beacons to be piggybacked on the data

\footnotetext{
${ }^{3}$ Results are signifi cantly worse for the greedy schemes when we investigate the number of packets instead of the amount of bytes, since beacon messages are generally much smaller than data packets.
}

packets. Furthermore, the transmission costs for the greedy scheme increases significantly with an increase in mobility. The better the available neighbor information due to a high beacon rate, the lower the increase in MAC overhead caused by increasing mobility. When mobility is high, a large fraction of the packets have to be sent multiple times because of the MAC callback. This ratio decreases when more accurate neighbor information is available, at the expense of an increase of the overhead caused by the beacons.

To analyze the transmission costs caused by the optimized greedy scheme in more detail, Figure 5 shows the specific components of the aggregate MAC traffic of Figure 4 for a beacon interval of 2 seconds.

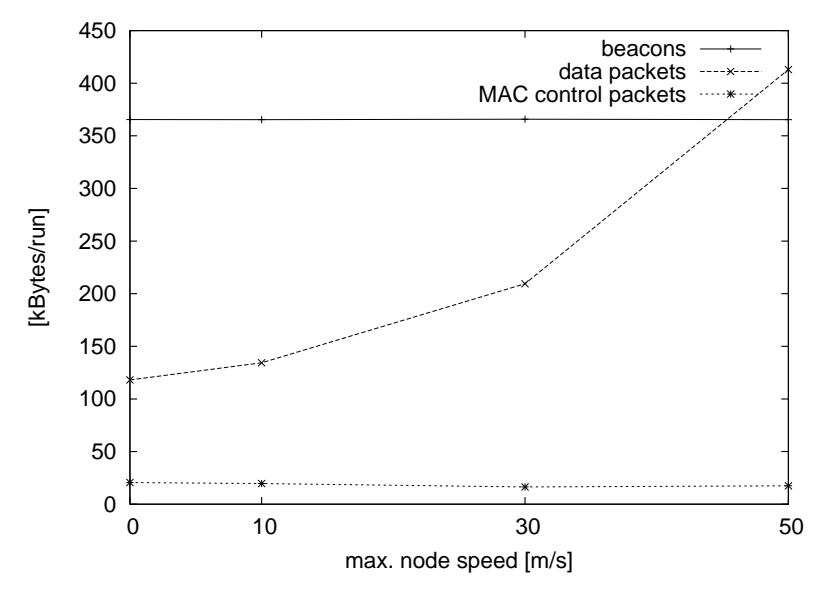

Fig. 5

COST COMPOSITION OF GREEDY OPT 2.0

The bandwidth consumed by beacon messages and MAC control packets (i.e., unicast acknowledgments of the data packets) is independent of the mobility rate. In contrast, the overhead caused by the transmission of data packets increases significantly with higher mobility. When comparing Figure 4 and 5, we observe that without mobility and excluding beacon traffic, the optimized greedy scheme consumes roughly as much bandwidth as area-based CBF. For a maximum node speed of $30 \mathrm{~m} / \mathrm{s}$, optimized greedy already consumes the same bandwidth as the active selection scheme (while the additional RTF/CTF messages in the active selection scheme also provide protection against the hidden terminal problem). For node speeds of $50 \mathrm{~m} / \mathrm{s}$ and above, the greedy scheme even significantly exceeds the bandwidth usage of the basic CBF scheme with its unsuppressed duplicates. At this node mobility, the forwarding overhead is higher than the overhead caused by the beacon messages of all 300 nodes and exceeds the forwarding overhead with no mobility almost by a factor of four.

With only one sender and receiver and a data rate of 4 $\mathrm{KBit} / \mathrm{s}$, the amount of data traffic is extremely low given the total number of nodes. At such low rates, the additional traffic caused by the optimized greedy scheme can be handled by the MAC layer without any problems. However, for reasonable 
combinations of beacon traffic and actual data traffic, we expect the overhead ratio to become much worse. When the additional traffic caused by repeated MAC callback results in congestion, data packets as well as beacon messages may be lost. The former have to be retransmitted at the cost of additional bandwidth consumption, while loss of the latter decrease the accuracy of the neighbor tables, further aggravating the MAC callback problem.

\section{CONCLUSIONS AND OUTLOOK}

The advantage of position-based routing over other ad-hoc routing protocols is the fact that nodes require only knowledge about the local neighborhood and the destination's location instead of global route topology. Therefore, position-based routing is better suited for networks with high mobility. With the contention-based forwarding mechanism proposed in this paper, even this local knowledge and hence the sending of beacon messages is no longer required. Any node with progress toward a destination can participate in the forwarding process without the need for this node to be registered in a neighbor table. For CBF, data packets are transmitted via single-hop broadcast. All nodes within radio range and with forward progress toward the destination are eligible to continue to forward the packet. Thus, the responsibility for the forwarding decision now lies with the set of possible next hops instead of the forwarding node, as is the case in conventional forwarding methods. Forwarding takes place after a contention period during which one or more nodes are selected as next hops. Selection of more than one next hop causes unwanted packet duplication. We presented different suppression strategies to avoid this.

For existing position-based forwarding schemes, node mobility results in frequent beacon messages to keep the neighbor tables reasonably up-to-date. Particularly for highly mobile networks, CBF can provide significant bandwidth savings through the elimination of beacon messages and the reduction of MAC layer retries for packet transmissions caused by inaccurate neighbor tables. Furthermore, the decrease in the total number of packets reduces the probability of packet collisions and inefficient routing caused by inaccurate neighbor tables is avoided.

The simulation results presented in this paper show that excessive re-sending of data due to outdated neighbor table entries as it is the case for traditional position-based routing can be completely avoided by the proposed contention-based forwarding approach. While one could argue that IEEE 802.11 is not a suitable MAC layer for high mobility simulations, the basic property of a very high dependency of routing performance on the accuracy of the neighbor table is shared by all conventional MAC protocols. Since CBF does not require any beaconing, and since CBF together with the area-based suppression strategy does not lead to any noticeable packet duplication, the resulting data volume overhead of the contentionbased method is much less than the data volume overhead generated with traditional position-based routing algorithms in highly mobile ad-hoc networks. Clearly, reducing load on the wireless medium is beneficial for ad-hoc networking in general. In the rare case where a packet duplication occurs due to $\mathrm{CBF}$, a simple strategy exists to improve the proposed suppression schemes: if duplication of packets occurs, these packets will be routed to the same destination at roughly the same time. Even with a very small state about which packets were recently forwarded, the duplicates can easily be suppressed in later nodes. Thus, packet duplication can be reduced while the simplicity of the suppression schemes is retained. In addition to the reduced forwarding overhead, the $\mathrm{CBF}$ schemes also provide a lower packet forwarding delay when node mobility is high. For the simulations, we used very conservative timer settings and we expect the reduction in forwarding delay to be much more pronounced with a well tuned CBF implementation.

One key item of future work will be the integration of CBF and MAC functionality. Since both serve a somewhat similar purpose their integration can significantly reduce the overhead incurred by the CBF scheme. In particular, we expect that it is possible to significantly reduce the runtime of the random timers used for the contention process. If a MAC layer with RTS/CTS is used to solve the hidden terminal problem (as is possible with IEEE 802.11), it can be combined with the RTF/CTF messages of active selection which will significantly increase the efficiency of this suppression strategy. Furthermore, a maximum response time $T$ which adapts to network load and node density can reduce the delay incurred by the contention period. So far, we have only considered greedy forwarding. In position-based routing, greedy forwarding fails if no neighbor with progress toward the destination exists. In such a case, a recovery strategy is used to circumnavigate the area with no reception. While Geodesic Anchors [3], as proposed for Terminodes routing, is directly applicable to $\mathrm{CBF}$ we also plan to investigate other alternatives that are more similar to the repair strategies of face- 2 and GPSR.

The use of directional antennas in ad-hoc networks recently gained increased scientific interest. This technology seems to be a promising candidate particularly in the context of areabased suppression.

\section{ACKNOWLEDGMENTS}

This work has been carried out within in the framework of the 'FleetNet' project as part of BMBF contract no. 01AK025D.

\section{REFERENCES}

[1] B. N. Karp and H. T. Kung, "GPSR: Greedy Perimeter Stateless Routing for Wireless Networks," in Proceedings of the sixth annual ACM/IEEE International Conference on Mobile computing and networking (MobiCom '00), Boston, Massachusetts, August 2000, pp. 243-254.

[2] S. Basagni, I. Chlamtac, V. R. Syrotiuk, and B. A. Woodward, "A Distance Routing Effect Algorithm for Mobility (DREAM)," in Proceedings of the fourth annual ACM/IEEE International Conference on Mobile computing and networking (MobiCom '98), Dallas, Texas, October 1998, pp. 76-84.

[3] L. Blažević, S. Giordano, and J.-Y. LeBoudec, 'Self-Organizing WideArea Routing," in Proceedings of SCI 2000/ISAS 2000, Orlando, July 2000. 
[4] P. Bose, P. Morin, I. Stojmenovic, and J. Urrutia, 'Routing with guaranteed delivery in ad hoc Wireless Networks," in Proceedings of the 3rd international workshop on Discrete algorithms and methods for mobile computing and communications (DIAL-M '99), Seattle, WS, August 1999, pp. 48-55.

[5] F. Kuhn, R. Wattenhofer, and A. Zollinger, "Worst-Case optimal and average-case effi cient geometric ad-hoc routing," in Proceedings of the fourth ACM international symposium on Mobile and ad hoc networking \& computing (MobiHoc '03), Annapolis, Maryland, June 2003, pp. 267278 .

[6] ANSI/IEEE Std 802.11, 1999th ed., ANSI/IEEE, 1999.

[7] L. Blažević, 'Scalable Routing Protocols with Applications to Mobility," Ph.D. dissertation, Swiss Federal Institute of Technology (EPFL), February 2002.

[8] M. Heissenbüttel and T. Braun, "A Novel Position-based and Beaconless Routing Algorithm for Mobile Ad-Hoc Networks," in Proc. of the 3rd IEEE Workshop on Applications and Services in Wireless Networks (ASWN '03), Bern, Switzerland, July 2003, pp. 197-209.

[9] H. Füßler, J. Widmer, M. Käsemann, M. Mauve, and H. Hartenstein, "Contention-Based Forwarding for Mobile Ad-Hoc Networks," to appear in Ad Hoc Networks, 2003.

[10] W. Gleißner and H. Zeitler, "The reuleaux triangle and its center of mass," Results in Mathematics, vol. 37, pp. 335 - 344, 2000.

[11] P. Karn, "MACA - A New Channel Access Method for Packet Radio," in Proceedings of the 9th ARRL/CRRL Amateur Radio Computer Networking Conference, September 1990, pp. 134-140.

[12] "The ns-2 network simulator," http://www.isi.edu/nsnam/ns/.

[13] D. B. Johnson and D. A. Maltz, 'Dynamic Source Routing in Ad Hoc Wireless Networks," in Mobile Computing, T. Imielinski and H. Korth, Eds. Kluwer Academic Publishers, 1996, vol. 353, pp. 153-181. 